\title{
Anticancer effect of caudatin in diethylnitrosamine-induced hepatocarcinogenesis in rats
}

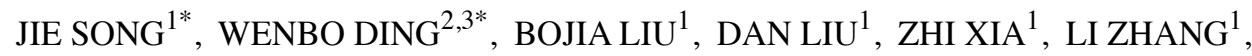 \\ $\mathrm{LI} \mathrm{CUI}^{1}$, YI LUO ${ }^{1}$, XIAOBIN JIA ${ }^{2}$ and LIANG FENG ${ }^{2}$

\begin{abstract}
${ }^{1}$ Department of Oncology, Affiliated Hospital of Integrated Traditional Chinese and Western Medicine, Nanjing University of Chinese Medicine, Nanjing, Jiangsu 210028; ${ }^{2}$ School of Traditional Chinese Pharmacy, China Pharmaceutical University, Nanjing, Jiangsu 211198; ${ }^{3}$ Ultrasonic Department, Affiliated Hospital of Integrated Traditional Chinese and Western Medicine, Nanjing University of Chinese Medicine, Nanjing, Jiangsu 210028, P.R. China
\end{abstract}

Received August 21, 2019; Accepted April 8, 2020

\section{DOI: $10.3892 / \mathrm{mmr} .2020 .11135$}

\begin{abstract}
An overwhelming endoplasmic reticulum stress (ERS) and the following unfolded protein response (UPR) can induce hepatic inflammation, fibrosis and hepatocellular carcinoma (HCC). Caudatin, one of the species of C-21 steroidal glycosides mainly isolated from the roots of Cynanchum bungei Decne, exhibits potent anticancer activities in vivo. However, the effect of caudatin on HCC remains unclear. In the present study, a diethylnitrosamine (DEN)-induced HCC model was established. Nodules and tumors in rat livers were monitored by T2-/T1-weighted-magnetic resonance imaging (MRI) using a $1.5 \mathrm{~T}$ scanner. Caudatin reduced the number and size of nodules and alleviated the inflammatory foci in the
\end{abstract}

Correspondence to: Professor Yi Luo, Department of Oncology, Affiliated Hospital of Integrated Traditional Chinese and Western Medicine, Nanjing University of Chinese Medicine, 100 Shizi Street, Hongshan Road, Nanjing, Jiangsu 210028, P.R. China

E-mail: robertluoyi@126.com

Professor Liang Feng, School of Traditional Chinese Pharmacy, China Pharmaceutical University, 639 Longmian Road, Nanjing, Jiangsu 211198, P.R. China

E-mail: wenmoxiushi@163.com

*Contributed equally

Abbreviations: ATF, activating transcription factor; CHOP, CCAAT/enhancer-binding protein homologous protein; DEN, diethylnitrosamine; eIF2 $\alpha$, eukaryotic initiation factor $2 \alpha$; ER, endoplasmic reticulum; ERDJ4, endoplasmic reticulum DnaJ homolog 4; GADD34, growth arrest and DNA damage-inducible protein; GRP78, glucose-regulated protein, $78 \mathrm{kDa}$; GRP94, glucose-regulated protein, $94 \mathrm{kDa}$; HCC, hepatocellular carcinoma; IRE1, inositol requiring enzyme 1; PERK, PKR-like endoplasmic reticulum kinase; PDIA4, protein disulfide-isomerase A4; UPR, unfolded protein response; $\mathrm{XBP} 1 \mathrm{~s}$, spliced $\mathrm{X}$-box-binding protein 1

Key words: caudatin, hepatocellular carcinoma, endoplasmic reticulum stress, glucose-regulated protein, $78 \mathrm{kDa}$, diethylnitrosamine liver. In addition, the hepatic pro-inflammatory levels of interleukin (IL) 6 , monocyte chemoattractant protein 1 and IL-1 $\beta$ were decreased in caudatin-treated rats. The DEN-induced surge in malondialdehyde, aspartate aminotransferase, alanine transaminase and TBIL were alleviated following caudatin treatment. The expression of ERS chaperones glucose-regulated protein, $94 \mathrm{kDa}$, glucose-regulated protein, $78 \mathrm{kDa}$ and protein disulfide-isomerase A4 and the proliferation marker $\mathrm{Ki}-67$ in liver nodules were all downregulated by caudatin as demonstrated by immunohistochemistry, reverse transcription-quantitative PCR and western blot analysis. Caudatin reduced the cytoprotective ERS sensor activating transcription factor 6-mediated signal transduction and inhibited the PKR-like endoplasmic reticulum kinase/eukaryotic initiation factor $2 \alpha$ activating transcription factor 4 pathway. However, the effect of caudatin on inositol requiring enzyme 1 signaling was negligible. In conclusion, restoration of the dysregulated UPR program was involved in the antitumor efficacy of caudatin without inducing cumulative hepatotoxicity.

\section{Introduction}

Hepatocellular carcinoma (HCC) is one of the most lethal and prevalent cancers, closely associated with cirrhosis and fibrosis with a variable etiology (1). Notably, its incidence and mortality are higher in developing countries, with an increasing burden on developed countries (2). Predominantly, HCC is caused by chronic hepatitis $\mathrm{B}$ and $\mathrm{C}$ viral infections, alcoholic liver and non-alcoholic fatty liver disease (3). Chronic inflammation promotes HCC progression (4). Furthermore, HCC is closely associated with immune suppression and tolerance (5). A great number of cytokines, immune-inhibitory receptors, pro-inflammatory cytokines and their ligands also prompt immunosuppression to contribute to HCC progression (6). Following viral hepatitis or chronic liver insults, elevated interleukin (IL) 6 activates hepatocyte proliferation, which ultimately results in $\mathrm{HCC}$ (7).

As a key cellular compartment, the endoplasmic reticulum (ER) is indispensable in protein synthesis and protein maturation, which needs the participation of folding enzymes and chaperones (8). Protein homeostasis is disturbed, with ER 
stress (ERS) occurring, when the amount of unfolded protein exceeds the ER capacity (9). ERS induction by infection has been implicated in HCC and disease progression with chronic inflammation via enhanced inflammation, oxidative stress-mediated DNA damage and hepatocyte proliferation. Notably, ERS is crucial for cancer cells to preserve malignancy and maintain resistance to therapy. Indeed, ERS participates in HCC radiation and chemotherapy resistance $(10,11)$. Furthermore, the escape of tumor cells from immunosurveillance has been attributed to ERS (9). ERS results in the gathering of misfolded or unfolded proteins. Under this situation, the unfolded protein response (UPR) in cells is activated to alleviate the superabundant protein load, including transient reduction of protein translation and misfolded protein degradation (8). In addition, folding enzymes and molecular chaperones strengthen the ER capability to fold and degrade more proteins (12).

Continuous UPR activation occurs in various types of cancer and is believed to facilitate oncogenic processes. The UPR can be activated by three ER sensors: Activating transcription factor-6 (ATF6), inositol-requiring enzyme 1 (IRE1) and protein kinase RNA-like endoplasmic reticulum kinase (PERK). The ER lumen houses several chaperones, including glucose-regulated protein-78 (GRP78)/binding immunoglobulin protein (BiP) and -94 (GRP94), and protein disulfide-isomerase A4 (PDIA4) (13). Molecular chaperones play an essential role in maintaining ER protein homeostasis. Normally, GRP78 is bound to the three ER sensors, maintaining them in an inactive form $(14,15)$. Upon dissociation from GRP78, PERK and IRE1 transform into oligomers or homodimers, activating the downstream pathways through autophosphorylation. Upon release by BiP, ATF6 is transformed into a transcription factor, stimulating the transcription of different ER chaperones (16,17). The effects of PERK and IRE1 are both proapoptotic and pro-survival, whereas the effect of ATF6 is principally cytoprotective (18). In human HCC, GRP78, ATF6, IRE1 and PERK expressions are higher than the basal levels and negatively associated with the overall survival and clinicopathological scores in HCC patients $(4,19)$.

Natural compounds exhibit promising applications in cancer therapy attributed to their special pharmacological activities and low toxicity (20). The roots of Cynanchum auriculatum Royle ex Wight, known as 'baishouwu' in China and in other Asian countries, have been widely used as a tonic supplement for strengthening kidney function in clinical settings (21). Caudatin has the highest antitumor capacity among several C-21 steroidal glycosides isolated from baishouwu, exhibiting selectivity towards hepatoma cell lines compared with other tumor cell lines (22). Furthermore, the inhibitory effect of caudatin has been validated in a H22 solid tumor model in vivo (20). Caudatin prevents tumor progression by stimulating DNA damage-mediated cell cycle arrest (23) or apoptosis (24). Previously, the present authors demonstrated that caudatin effectively inhibited human hepatoma cell growth and metastasis (25). However, the in vivo effect of caudatin in the orthotopic tumor model has not yet been elucidated. Therefore, the present study used the diethylnitrosamine (DEN)-induced cirrhotic rat model with HCC to test the safety and antitumor efficacy of caudatin and explore the mechanism of action.

\section{Materials and methods}

Reagents and materials. Caudatin, Tween-20, bovine serum albumin and sodium dodecyl sulfate were purchased from Sigma-Aldrich Shanghai Trading Co. Ltd. The various antibodies used were: ATF4 (cat. no. sc-390063) were obtained from Santa Cruz Biotechnology, Inc. eIF2 $\alpha$ (cat. no. 5324), phosphorylated (p)-eIF2 $\alpha$ (cat.no. 3398), GRP78 (cat. no. 3183), IRE1 $\alpha$ (cat. no. 3294), p-PERK (cat. no. 3179) and PERK (cat. no. 3192) were purchased from Cell Signaling Technology, Inc. Tubulin (cat. no. ab7291), p-IRE1 $\alpha$ (cat. no. ab48187), Ki67 (cat. no. ab16667), ATF6 (cat. no. ab203119), GAPDH (cat. no. ab181602), PDIA4 (cat. no. ab82587) and GRP94 (cat. no. ab2791) were purchased from Abcam. All other chemicals were of analytical grade and were obtained commercially.

Diethylnitrosamine-induced HCC rat model. A total of 18 female Sprague-Dawley rats (age, 2 months; weight, $200 \pm 20 \mathrm{~g}$ ) were obtained from Shanghai Lab Animal Research Center. Rats were maintained on a standard diet and water ad libitum (12 h light/dark cycle with humidity of $60 \pm 5 \%$ and temperature $22 \pm 3{ }^{\circ} \mathrm{C}$ ). Rats were intraperitoneally injected with $70 \mathrm{mg} / \mathrm{kg}$ of diethylnitrosamine (DEN; Sigma-Aldrich, Merck KGaA) once per week for 10 continuous weeks. All animal experiments were approved by the Institutional Animal Care and Use Committee of Jiangsu Provincial Academy of Chinese Medicine (approval no. AEWC-20170727-05). The 18 rats were divided into three groups and received treatment from week 6-20, with six rats in each group: DEN-treated control group, and low and high doses of Caudatin groups (25 or $50 \mathrm{mg} / \mathrm{kg}$, respectively; oral administration), 6 days oral administration per week. Rats were sacrificed 10 weeks following the last DEN injection. Normal rats were used as the blank group.

Biochemical assays. Prior to sacrifice, the blood of rats was collected and centrifuged at $1,411 \mathrm{x} \mathrm{g}$ for $10 \mathrm{~min}$ at $4^{\circ} \mathrm{C}$ to measure serum aspartate aminotransferase (AST), alanine transaminase (ALT) and total bilirubin (TBIL) using an autoanalyzer (Type 7020, Hitachi, Ltd.). The supernatant of liver homogenates was used for the measurements of malondialdehyde (MDA). MDA was determined spectrophotometrically at $535 \mathrm{~nm}$. Intracellular cytokine levels were monitored in liver whole-cell lysate using IL-6 (cat. no.431307), IL-1 $\beta$ (cat. no. 437007), monocyte chemoattractant protein-1 (MCP-1; cat. no. 438807) and tumor necrosis factor (TNF) $\alpha$ (cat. no. 438207) ELISA kits (Biolegend, Inc.).

MRI. Rats were anesthetized with $2 \%$ isoflurane during MRI observation in a wrist coil. A supine position was scanned using a 1.5 T MRI scanner (Echo speed; GE Healthcare). T1-weighted, T2-weighted and diffusion-weighted imaging (DWI) sequences were performed. Rats were injected with Magnevist (Schering; Bayer HealthCare Pharmaceuticals) through the tail vein and contrast-enhanced MR scanning (T1CE) was performed following injection.

Immunohistochemistry and hematoxylin and eosin $(H \& E)$ staining. Livers were excised and, following $48 \mathrm{~h}$ fixation in paraformaldehyde at room temperature, and paraffin sections 


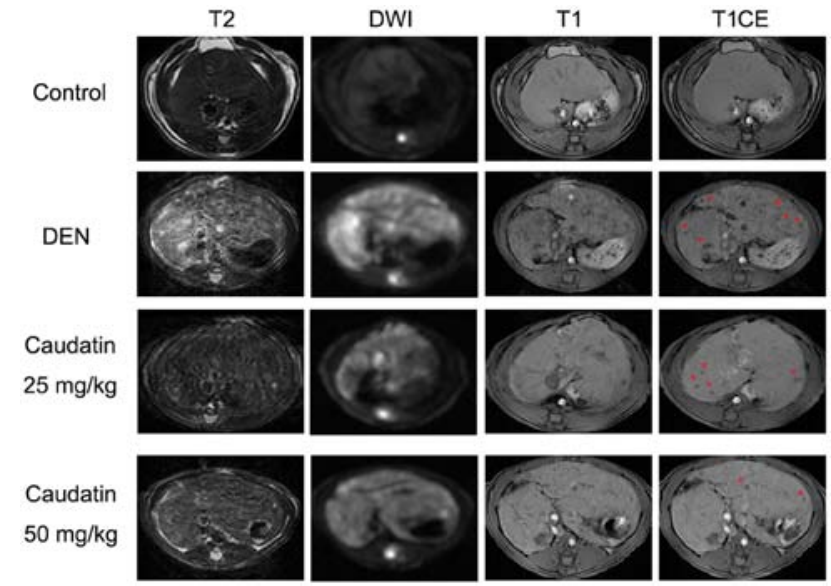

Figure 1. In vivo MRI images of liver tumors in rats. Representative images of tumors from each group. T2- and DWI-weighted MR images of the liver, as well as T1CE MRI following injection of Magnevist ( $n=6 /$ group). Tumors in T1CE MRI are indicated with red asterisks. MRI, magnetic resonance imaging; DWI, diffusion-weighted imaging; T1CE, tail vein and contrast enhanced.

(thickness, 4- $\mu \mathrm{m}$ ) were prepared. Sections were deparaffinized by rinsing twice in xylene for $10 \mathrm{~min}$ each. The tissue sections were hydrated with ethanol series (100, 95, 75 and 50\%) and washed in distilled water. Heat-induced epitope retrieval was achieved with Tris-EDTA ( $\mathrm{pH} \mathrm{8).} \mathrm{Endogenous} \mathrm{peroxidase}$ activity was quenched by $3 \% \mathrm{H}_{2} \mathrm{O}_{2}$ and incubated with $5 \%$ normal goat serum (cat. no. 7481; Abcam) at room temperature for $30 \mathrm{~min}$, followed by overnight incubation with anti-Ki-67 (1:500), anti-GRP78 (1:200) antibody at $4^{\circ} \mathrm{C}$. Sections were then washed with PBS and incubated with goat anti-rabbit IgG H\&L (horseradish peroxidase) antibodies (cat. no. 205718; $1: 2,000$; Abcam) at $37^{\circ} \mathrm{C}$ for $30 \mathrm{~min}$. Immunoreactivity was identified as brown in liver sections counterstained with hematoxylin.

The deparaffinized sections (thickness, 4- $\mu \mathrm{m}$ ) was also stained with H\&E kits (Servicebio, Inc.) according to the manufacturer's instructions. Sections were stained with hematoxylin at room temperature for $5 \mathrm{~min}$, followed by $0.5 \%$ eosin staining at room temperature for $3 \mathrm{~min}$. The H\&E staining was independently inspected by a pathologist in a blinded manner. The length of the scale bar is given in the figure legends.

RNA isolation and reverse transcription-quantitative $(R T-q)$ $P C R$. RNA was extracted from liver tumors or normal livers with TRIzol (Invitrogen; Thermo Fisher Scientific, Inc.). RNA extraction, cDNA synthesis and qPCR were performed according to the manufacturer's protocols. Initially, the RNA samples were screened based on their purity $(260 / 280 \geq 1.8)$ with a NanoDrop spectrophotometer (Thermo Fisher Scientific, Inc.). cDNA was synthesized via Superscript III, using $5 \mu \mathrm{g}$ of RNA and oligo-dT as primer. RT-qPCR was performed on an ABI 7900HT real-time PCR instrument (Applied Biosystems; Thermo Fisher Scientific, Inc.) with primers as follows: GAPDH (NM_017008.4,5'-AGTGCCAGCCTCGTCTCATA-3',5'-TAC GGCCAAATCCGTTCACA-3'), GRP78 (NM_013083.2, 5'-TCGACTTGGGGACCACCTAT-3', 5'-GCGGCCGTT CTTGAATACAC-3'), GRP94 (NM_001012197.2, 5'-TAA GCTCTATGTGCGCCGAG-3'，5'-TCACGGGAAACA
TTGAGGGG-3'), ATF4 (NM_024403.2, 5'-TTAAGCCAT GGCGCTCTTCA-3'，5'-GACATTAAGTCCCCCGCC AA-3'), PDIA4 (NM_053849.1, 5'-AGTGGAGAGGAC GTCAATGC-3', 5'-CCCTGACTGGTCCCTTGTTG-3'), ERDJ4 (NM_012699.2, 5'-AACAGGACGAAGGTTGCT CG-3', 5'-AACTGACTGTGGAGTTGCCA-3') and GADD34 (NM_133546.3, 5'-GAGAATGTGGCCCCAGTTGA-3', 5'-ACAATGCTGGGTACTCTGGC-3'). The cycling conditions were as follows: Initial denaturation at $95^{\circ} \mathrm{C}$ for $30 \mathrm{sec}$, followed by 40 cycles of primer annealing at $60^{\circ} \mathrm{C}$ for $30 \mathrm{sec}$ and an extension of amplicon at $72^{\circ} \mathrm{C}$ for $1 \mathrm{~min}$. The RNA levels were quantified using the $2^{-\Delta \Delta \mathrm{Cq}}$ method (26).

Western blot analysis. Protein extraction was performed by homogenizing the rat liver in ice-cold hypotonic buffer containing PMSF and Nonidet P-40. Total protein was quantified, mixed with sample buffer and boiled at $90^{\circ} \mathrm{C}$ for $5 \mathrm{~min}$. The protein concentration was determined with a bicinchoninic acid kit (Beyotime Institute of Biotechnology). Equal amount of protein $(30 \mu \mathrm{g})$ was separated by electrophoresis in $12 \%$ SDS-PAGE, transferred to PVDF membranes. After blocking with $5 \%$ non-fat milk in TBST $(0.1 \%$ Tween-20) for $1 \mathrm{~h}$ at room temperature, the membranes were incubated with antibodies against ATF4 (1:1,000), eIF2 $\alpha$ (1:500), p-eIF2 $\alpha$ (1:500), GRP78 (1:1,000), IRE1 $\alpha$ (1:1,000), p-PERK (1:500), PERK $(1: 1,000)$, Tubulin $(1: 2,000)$, p-IREl $\alpha$ (1:500), Ki67 (1:2,000), ATF6 (1:2,000), GAPDH (1:5,000), PDIA4 (1:1,000) or GRP94 $(1: 1,000)$ overnight at $4^{\circ} \mathrm{C}$. All the antibodies were diluted in $5 \%$ non-fat milk in TBST. Slides were then incubated with horseradish peroxidase-conjugated goat anti-rabbit IgG H\&L (cat. no. 205718; 1:2,000; Abcam) or goat anti-rat IgG H\&L (cat. no. 97057; 1:2,000; Abcam) secondary antibodies for $2 \mathrm{~h}$ at room temperature. The immune complexes were visualized with an enhanced chemiluminescence detection kit (GE Healthcare Life Sciences). The quantification of protein expression was normalized with control protein expression. Band intensity quantification was performed using ImageQuant TL software (version 7.0; GE Healthcare Life Sciences).

Statistical analysis. Assays were conducted in three independent experiments. Statistical comparisons were performed using one-way analysis of variance followed by Tukey's multiple comparison post hoc test using GraphPad Prism 5.0 software (GraphPad Software, Inc.). $\mathrm{P}<0.05$ was considered to indicate a statistically significant difference.

\section{Results}

Caudatin inhibited HCC development in rats. The therapeutic effect of caudatin in DEN-induced HCC rats was assessed using MRI. The liver tumors appeared homogeneously hypoor isointense in T1-weighted MR images and hyperintense in DWI and T2 sequence images relative to the adjacent normal liver (Fig. 1). The liver tumors appeared iso- or slightly hypo-intense on T1CE images, as indicated by the red asterisk in Fig. 1. DEN-induced rats demonstrated a higher number of tumor nodules in the liver as observed in the MRI analysis. Tumor nodules were considerably inhibited in the caudatin group compared with the model rats, particularly in the $50 \mathrm{mg} / \mathrm{kg}$ treatment group. 


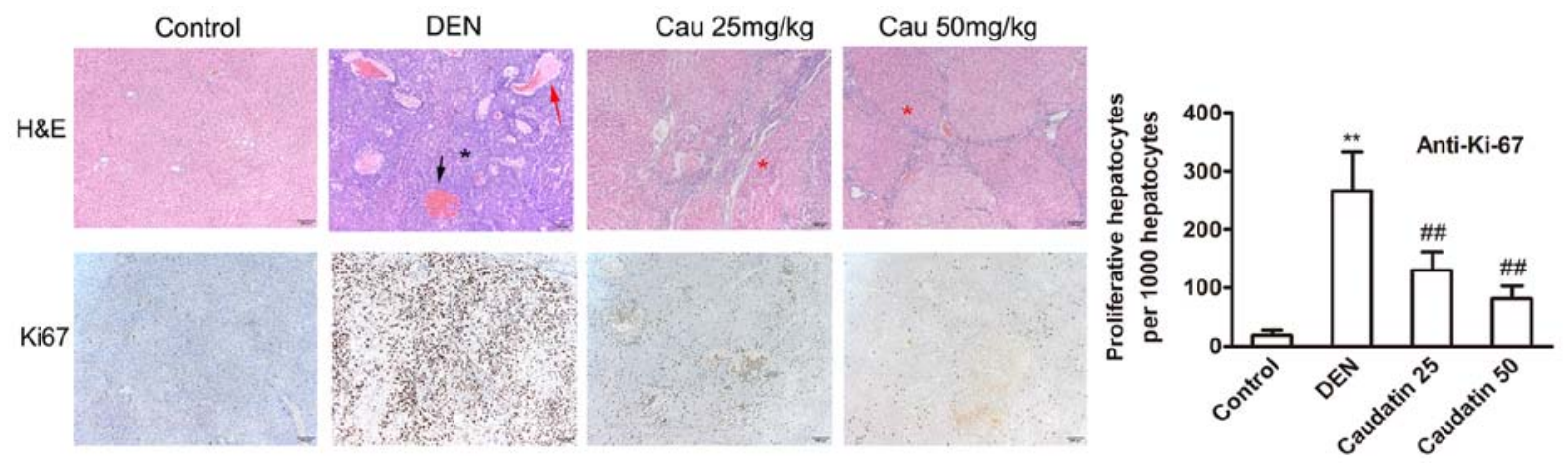

Figure 2. H\&E staining and immunohistochemical analysis of Ki-67 in liver sections. Hemorrhage (black arrow), hepatic sinus dilation (red arrow), tumor cells (black asterisk) and necrosis are evident in the DEN group. Fibrosis (red asterisk) is observed in the caudatin-treated group. Hepatocytes stained positive with the proliferation marker (Ki-67) were counted under a microscope at x100 magnification. Graph shows quantitation of Ki-67 immunohistochemistry. ${ }^{* *} \mathrm{P}<0.01$ vs. control; ${ }^{\#} \mathrm{P}<0.01$ vs. DEN group ( $\mathrm{n}=6 /$ group). The $\mathrm{Ki}-67$ intensity is reduced in caudatin-treated groups compared with the DEN group as shown in the immunohistochemical analysis (scale bar=100 $\mu \mathrm{m}$ ). H\&E, hematoxylin and eosin staining; DEN, diethylnitrosamine.

DEN-initiation resulted in multiple and visible surface liver tumors (data not shown). In histological staining during end-point necropsy, histopathology examinations further verified the observations of MR imaging. The DEN-induced tumors were either well, moderate, or poorly differentiated HCC, confirming the progress of HCC as a result of the DEN insult (Figs. 2 and S1). At week 20, tumor nodules were observed in a background of fibrosis (Figs. 2 and S1). Additionally, hemorrhage, adenoid structures and necrosis were observed in the DEN group. Furthermore, pseudoadenoid structures were formed by necrosis of central tumors (Fig. S1). In the caudatin group, tumor necrosis was attenuated and the number of cancerous cells reduced; however, fibrosis, hepatic sinus dilation and infiltration of inflammatory cells were still obvious (Fig. 2). Caudatin also inhibited the proliferation marker Ki-67 (Fig. 2). Food intake by weight was similar among the four groups and caudatin demonstrated no significant effect on body weight (Table I). Notably, caudatin substantially reduced HCC incidence, macroscopic nodules and hepatic preneoplastic lesions that may advance into tumors (Table I).

Effect of caudatin on biochemical markers of hepatic injury. The serum levels of TBIL, AST, ALT and malondialdehyde MDA were significantly elevated in the liver homogenates following DEN treatment (Table II; $\mathrm{P}<0.05$ ). ALT was elevated to $30.65 \pm 2.02$ in DEN-treated rats and reduced to $18.61 \pm 0.85$ in caudatin-treated rats $(50 \mathrm{mg} / \mathrm{kg})(\mathrm{P}<0.05)$, compared with the normal group. The base level of ALT in the control group was $10.85 \pm 1.01 \mathrm{U} / \mathrm{ml}$. Simultaneously, AST increased in DEN rats $(36.04 \pm 2.48)$ and markedly decreased to $23.10 \pm 1.18 \mathrm{U} / \mathrm{ml}$ in caudatin-treated rats $(\mathrm{P}<0.05)$. TBIL increased to $1.02 \pm 0.18$ in the DEN group (vs. $0.27 \pm 0.08$, control) but was reduced substantially $(0.77 \pm 0.19)$ in rats administered caudatin following DEN $(\mathrm{P}<0.05)$. In the DEN-induced rats, the MDA level was $528.18 \pm 58.42 \mathrm{~mol} / \mathrm{g}$ in liver tissues and reduced to $370.19 \pm 32.57 \mathrm{~mol} / \mathrm{g}$ in the caudatin-treated group $(\mathrm{P}<0.05)$. The prevention of ALT, AST and TBIL leakage from the liver suggested a potential protective effect of caudatin in DEN-induced liver damage.

Caudatin-mediated repression in hepatic tumorigenesis is associated with reduced hepatic pro-inflammatory cytokines.
Numerous animal studies have suggested that DEN-promoted liver tumorigenesis is associated with an amplified pro-inflammatory response (27-29). In the present study, the infiltration of inflammatory cells was demonstrated in the H\&E staining of the liver (Fig. 2). Consistently, the tissue protein levels of pro-inflammatory cytokines, including IL- 6 , TNF- $\alpha$, MCP-1 and IL-1 $\beta$, were elevated in the DEN group compared with the control group (Fig. 3). IL-6, MCP-1 and IL-1 $\beta$ were reduced in caudatin-treated rats $(\mathrm{P}<0.05)$.

Effect of caudatin on chaperone expression in the HCC model. Reportedly, DEN-induced hepatocarcinogenesis induces ERS (13). Therefore, the expression of the (co-) chaperones GRP94, GRP78 and PDIA4 in liver nodules was examined. Based on immunohistochemical analysis, it was observed that the basal level of pro-survival GRP78 in non-HCC livers was markedly low. Notably, a considerable amount of GRP78 was observed in the liver nodules of the DEN group (Fig. 4). However, an inhomogeneous pattern of GRP78-positive HCC cells was observed within the nodules, with only a few GRP78-positive cells in the surrounding tissue. The protein and mRNA level of GRP78 was reduced following caudatin treatment (Fig. 4). Additionally, both mRNA and protein levels of GRP94 and PDIA3 followed a similar pattern.

Effect of caudatin on the expression of ER sensors and UPR targets in DEN-induced liver tumors. Next, the effects of caudatin on the pattern of UPR signaling in liver nodules was evaluated. First, the deactivation of UPR targets was examined at the protein level. Reportedly, the PERK-eIF2 $\alpha$-ATF4 pathway is activated during hepatocarcinogenesis (30). p-PERK mediates the phosphorylation of the eIF2a, p-eIF2a subsequently activates ATF4 and ATF4 upregulates the transcription of growth arrest and DNA-damage-inducible protein 34 (GADD34) (31). In the present study, DEN markedly activated the PERK-eIF2 $\alpha$-ATF4 pathway, as indicated by the significantly enhanced expression of p-PERK and p-eIF2 $\alpha$ and downstream signaling molecules (ATF4 and GADD34). Consistent with the transcriptional suppression, caudatin inhibited the ATF4 protein levels (Fig. 5A). Furthermore, caudatin repressed ATF4 and GADD34 transcription (Fig. 5B). 
Table I. Effect of caudatin on the body weight, food consumption and the number of macroscopic hepatocyte nodules.

\begin{tabular}{lcccc}
\hline & \multicolumn{3}{c}{ Groups } \\
\cline { 2 - 5 } Variable & Control & DEN & Caudatin $25 \mathrm{mg} / \mathrm{kg}$ & Caudatin $50 \mathrm{mg} / \mathrm{kg}$ \\
\hline Final body weight, $\mathrm{n}$ & $559.4 \pm 19.5$ & $400.1 \pm 37.2^{\mathrm{a}}$ & $414.2 \pm 39.3$ & $447.3 \pm 49.7$ \\
Food consumption, g/d & $2.9 \pm 0.2$ & $3.0 \pm 0.2$ & $2.9 \pm 0.1$ & $3.0 \pm 0.2$ \\
Total no. of nodules & 0 & 78 & 55 & 45 \\
Mean measurement of nodule & 0 & $13 \pm 2^{\mathrm{a}}$ & $9 \pm 1^{\mathrm{b}}$ & $7 \pm 1^{\mathrm{b}}$ \\
$>3 \mathrm{~mm}$ & 0 & 39 & 22 & 15 \\
$<3$ to $>1$ mm & 0 & 19 & 18 & 12 \\
$<1 \mathrm{~mm}$ & 0 & 20 & 15 & 18
\end{tabular}

Each value is expressed as mean \pm standard deviation ( $\mathrm{n}=6 /$ group). ${ }^{\mathrm{a}} \mathrm{P}<0.05$ vs. control, ${ }^{\mathrm{b}} \mathrm{P}<0.05$ vs. DEN. DEN, diethylnitrosamine.

Table II. The effect of caudatin on biochemical markers of hepatic injury in DEN rats.

\begin{tabular}{lllll}
\hline Treatment & ALT, U/ml & AST, U/ml & TBIL, mg/dl & MDA, mol/g tissue \\
\hline Control & $10.85 \pm 1.01$ & $14.75 \pm 0.71$ & $0.27 \pm 0.08$ & $336.70 \pm 23.98$ \\
DEN & $30.65 \pm 2.02^{\mathrm{a}}$ & $36.04 \pm 2.48^{\mathrm{a}}$ & $1.02 \pm 0.18^{\mathrm{a}}$ & $528.18 \pm 58.42^{\mathrm{a}}$ \\
Caudatin $25 \mathrm{mg} / \mathrm{kg}$ & $27.29 \pm 1.60^{\mathrm{b}}$ & $28.35 \pm 1.18^{\mathrm{b}}$ & $0.83 \pm 0.11$ & $436.79 \pm 22.97^{\mathrm{b}}$ \\
Caudatin $50 \mathrm{mg} / \mathrm{kg}$ & $18.61 \pm 0.85^{\mathrm{b}}$ & $23.10 \pm 1.18^{\mathrm{b}}$ & $0.77 \pm 0.19^{\mathrm{b}}$ & $370.19 \pm 32.57^{\mathrm{b}}$ \\
\hline
\end{tabular}

Each value is expressed as mean \pm standard deviation ( $\mathrm{n}=6 /$ group). ${ }^{\mathrm{a}} \mathrm{P}<0.01 \mathrm{vs}$. control, ${ }^{\mathrm{b}} \mathrm{P}<0.05$ vs. DEN. DEN, diethylnitrosamine; ALT, alanine transaminase; AST, aspartate aminotransferase; TBIL, total bilirubin; MDA, malondialdehyde.

In addition, the phosphorylation of PERK and eIF2a was inhibited following caudatin treatment.

Western blotting revealed a robust cleavage of ATF6 in liver nodules compared with the mild expression of cleaved-ATF6 observed in saline-treated rat livers, indicating that the ATF6 pathway was activated at week 20 (Fig. 5A). Caudatin treatment reduced ATF6 cleavage. The reduced ratio of full ATF6/cleaved ATF6 protein expression was clearly upregulated following caudatin treatment.

Next, the IRE1 pathway was investigated. IRE1 activation promotes X-box binding protein 1 (XBP1) mRNA splicing to generate a more active spliced XBP1 (XBP1s), inducing genes involved in protein folding, such as endoplasmic reticulum DnaJ homolog 4 (ERDJ4) (32). Notably, the ratios of phosphorylated IRE1 to total IRE1 levels were higher in liver nodules than normal liver tissues. However, caudatin failed to impact the expression of phosphorylated IRE1. Consistently, the RT-qPCR analyses revealed a non-significant decrease in the mRNA level of the XBP-1 specific target gene, ERDJ4 (33) (Fig. 5B). In summary, these results indicated that the DEN-triggered excessive UPR in rats was suppressed by caudatin, probably through inhibition of the ATF6 and PERK-eIF2 $\alpha$-ATF4 pathways, but not the IRE1 pathway.

\section{Discussion}

In the present study, caudatin markedly decreased the incidence of HCC, and reduced macroscopic nodules and hepatic preneoplastic lesions in the DEN-induced HCC model. DEN, a widely used hepatocarcinogen, affects cancer initiation by inducing the formation of DNA-strand breaks and carcinogen adducts, thereby resulting in hepatocarcinogenesis (34). Reactive oxygen species (ROS) and ERS have been demonstrated to play a role in DEN-induced hepatocellular carcinogenesis (35). Oxidative stress can be enforced by ERS (36). Furthermore, nonalcoholic steatohepatitis, hepatosteatosis and viral hepatitis also increase the risk of HCC. ERS is involved in the pathogenesis of these disorders (37,38). MDA, ALT, AST and TBIL are valuable markers, widely used in animal studies to diagnose and observe the progress of hepatocarcinogenesis $(39,40)$. Consistently, the values of previously mentioned parameters increased sharply in the DEN-group as compared with the normal control group (Table II) in the present study. Normalization of elevated TBIL, MDA, AST and ALT release from the liver proposes a potent protective effect of caudatin against DEN-induced liver damage.

ER provides a quality control system, regulating the modification and folding of membrane and secretory proteins and eliminating misfolded polypeptides through autophagic degradation or ER-associated degradation (41). A variety of toxic insults including $\mathrm{Ca}^{2+}$ overload, failure of protein folding, synthesis, degradation or transport and hypoxia can interrupt the ER function and give rise to ERS (42). The accumulation of misfolded and unfolded proteins in the ER is denoted as ERS $(43,44)$. Tumors frequently yield increased mutant proteins, 

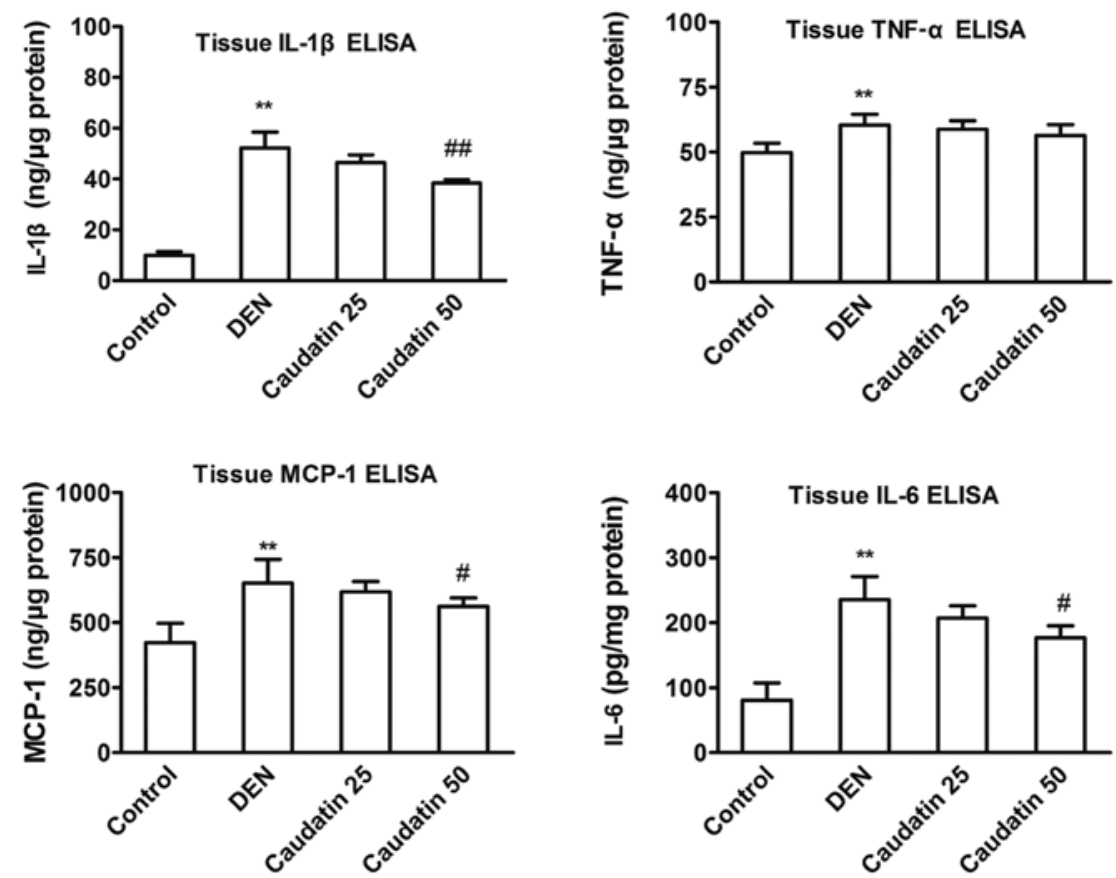

Figure 3. Effects of caudatin administration on hepatic pro-inflammatory cytokines. IL-6, TNF- $\alpha$, MCP-1 and IL-1 $\beta$ levels of liver tissues were examined by ELISA kits ( $\mathrm{n}=6$ /group). ${ }^{* *} \mathrm{P}<0.01$ vs. control; ${ }^{*} \mathrm{P}<0.05,{ }^{\# \#} \mathrm{P}<0.01$ vs. DEN group. IL, interleukin; TNF, tumor necrosis factor; MCP-1, monocyte chemoattractant protein 1; DEN, diethylnitrosamine.

A

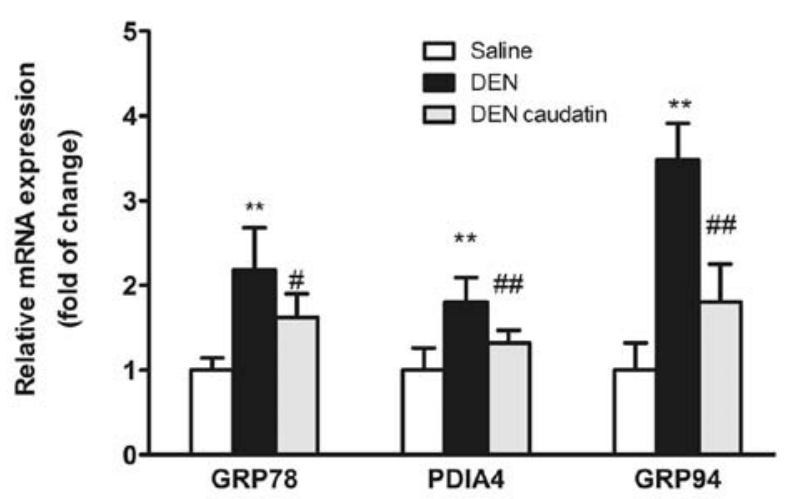

C

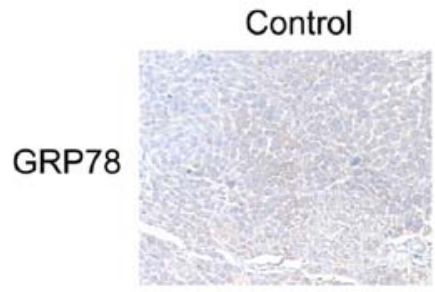

B
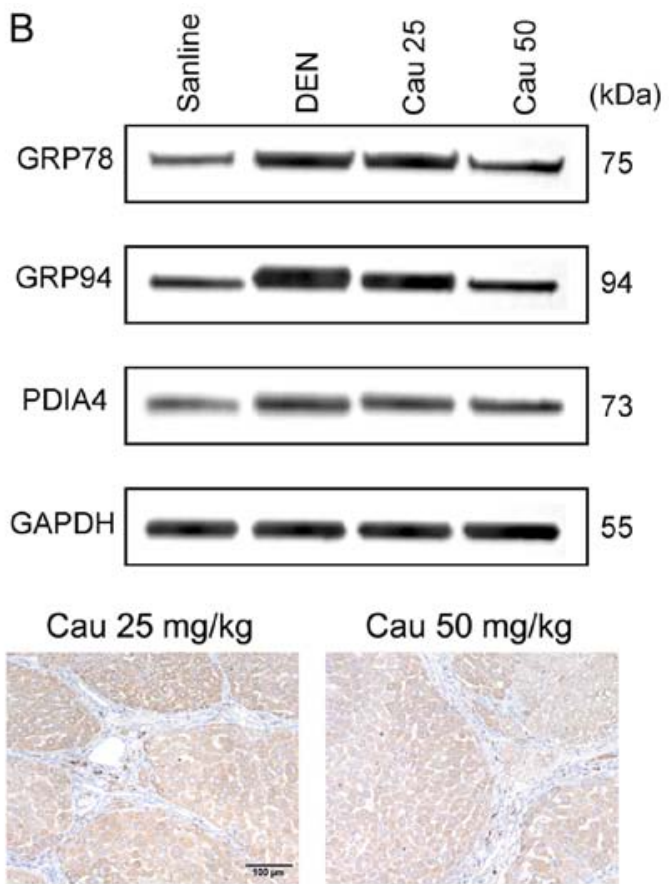

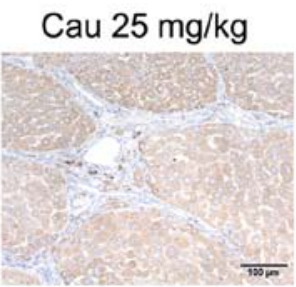

Cau $50 \mathrm{mg} / \mathrm{kg}$

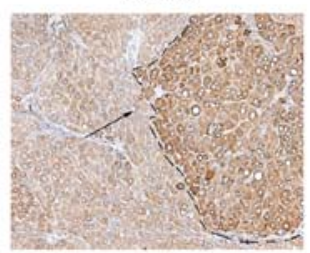

Figure 4. Effect of caudatin on the expression of chaperones in the DEN-induced HCC model. (A) Reverse transcription-quantitative PCR of GRP78, GRP94 and PDIA4 following the caudatin $\left(50 \mathrm{mg} / \mathrm{kg}\right.$ ) treatment. ${ }^{* * *} \mathrm{P}<0.01$ vs. control; ${ }^{*} \mathrm{P}<0.05,{ }^{* \#} \mathrm{P}<0.01$ vs. DEN group. (B) Western blot analysis of chaperone proteins. Results are representative of three independent experiments. (C) GRP78 immunostaining in the liver. Arrows indicate tumors (scale bar=100 $\mu \mathrm{m}$ ). DEN, diethylnitrosamine; HCC, hepatocellular carcinoma; GRP78, glucose-regulated protein, 78 kDa; GRP94, glucose-regulated protein, 94 kDa; PDIA4, protein disulfide-isomerase A4.

beyond the normal ER capacity, with the vascular supply ultimately failing to meet the nutrient demands (45). The UPR reinstates protein folding homeostasis and alleviates ERS by decreasing protein synthesis, facilitating protein degradation, augmenting protein folding and enhancing lipid synthesis (46).
Additionally, the UPR exerts an essential role in cancer cells maintaining malignancy and therapy resistance (47). Compared with normal tissues, sustained UPR activation has been described in numerous solid tumor types, including HCC liver tissues (48). 

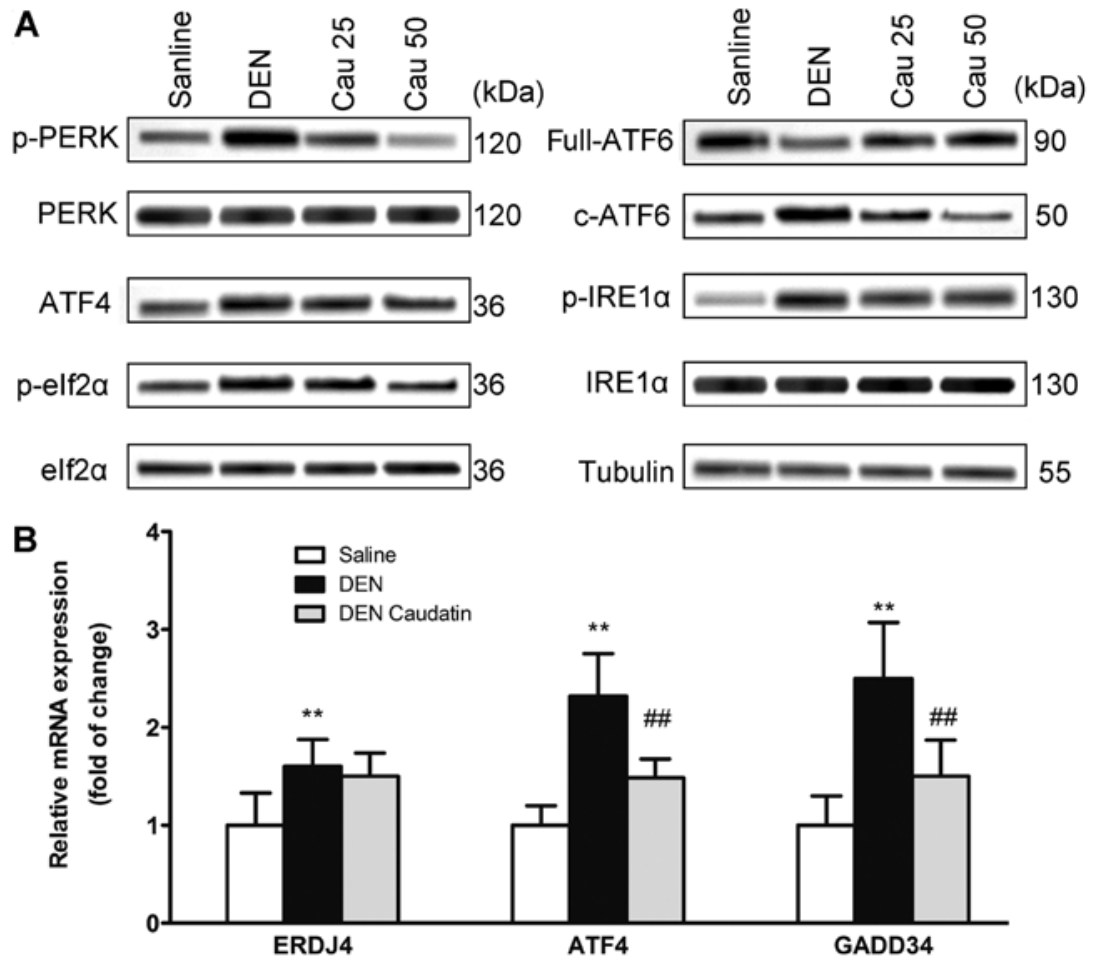

Figure 5. Effect of caudatin on the expression of IRE1 $\alpha$ and ATF6 and activation of the PERK/eIF2 $\alpha / A T F 4$ pathway in the DEN-induced HCC model. (A) Representative western blot analysis of ERS protein expression. (B) Reverse transcription-quantitative PCR of ERDJ4, ATF4, GADD34 following caudatin $(50 \mathrm{mg} / \mathrm{kg})$ treatment. ${ }^{* *} \mathrm{P}<0.01$ vs. control, ${ }^{\# \#} \mathrm{P}<0.01$ vs. DEN group. IRE1, inositol requiring enzyme 1 ; ATF, activating transcription factor; PERK, PKR-like endoplasmic reticulum kinase; eIF2 $\alpha$, eukaryotic initiation factor $2 \alpha$; DEN, diethylnitrosamine; HCC, hepatocellular carcinoma; ERDJ4, endoplasmic reticulum DnaJ homolog 4; GADD34, growth arrest and DNA damage-inducible protein; p-, phosphorylated.

The major chaperone, GRP78, is a dominant supervisor of the UPR (49). Generally, GRP78 is associated with a greater risk of cancer recurrence and poor outcomes. GRP78 can impede pro-apoptotic and caspase-4 pathways, stimulating metastasis and therefore yielding a worse prognosis (50). In some toxicology/pharmacokinetic studies in patients and monkeys, anti-GRP78 antibodies were well tolerated $(51,52)$. Furthermore, despite the partial GRP78 levels following treatment with anti-GRP78 agents, the adult liver can function normally, indicating that the anti-GRP78 damage to the normal liver may be limited (51). In the present study, the base liver levels of pro-survival GRP78 were relatively low and DEN treatment induced a marked amount of GRP78 in the liver tumors. In immunohistochemical staining, the number of GRP78-positive cells were considerably reduced by caudatin treatment. This result was further confirmed by RT-qPCR and western blot analysis. A similar tendency was observed with other chaperones including GRP94 and PDIA4.

ERS stimulates exosome release in PERK- and IRE1 $\alpha$-dependent manners (53). A rapid increase in ER-related proteins, such as ATF6 and spliced XBP1, is observed in patients with severe liver fibrosis or with $\mathrm{HCC}$, as well as in $\mathrm{CCl}_{4}$-induced fibrotic mouse liver tissues $(19,54,55)$. Consistently, both IRE1 and ATF6, as well as the PERK arms of the UPR, exhibited notable tumor-specific activation in the present study. ATF6 cleavage was elevated in the DEN-induced rat liver nodules compared with the saline group at week 20. The DEN-induced activation of ERS sensors, the PERK pathway and ATF6 cleavage, were evidently reduced by caudatin treatment. Nevertheless, the effect of caudatin on the ratio of phosphorylated IRE1 $\alpha /$ total IRE1 $\alpha$ and on the transcription of the downstream target, ERDJ4, was minimal. IRE1 is the most conserved transducer of the UPR, a surveillance mechanism that guarantees homeostasis in the eukaryotic ER. Notably, PERK mediates the cell cycle exit during the mammalian UPR (56). IRE1 is activated by the binding to unfolded proteins or separation from the suppressive interaction with chaperone GRP78; ultimately, IRE1 catalyzes the XBP1 transcript (57). XBP1 mRNA further splices into XBP1s, which participates in the protein folding of ERDJ4. In addition, GRP78 overexpression coupled with ERDJ4 shrinks the induction of CHOP in ERS and decreases ERS-induced apoptosis (58).

It has been reported that excessive or sustained activation of the UPR results in chronic inflammation (59). The transmission of ERS to macrophages promotes the inflammatory response in the HCC microenvironment (60). Additionally, cytokines can augment ERS in a positive feedback manner and encourage tumor growth (61). IL-6 is one of the best characterized tumorigenic, inflammatory cytokines, especially stimulating the development of $\operatorname{HCC}(62,63)$. Elevated IL-6 levels have been observed in both HCC and liver cirrhosis (64). Even though IL-6 is predominantly secreted by resident immune cells, hepatocy tes contribute to the total IL-6 expression in the liver microenvironment (7). In turn, IL-6 hastens compensatory hepatocyte proliferation, principally through tumor progression (65). In the present study, liver inflammatory foci and hepatic pro-inflammatory biomarkers, including IL-6, MCP-1 and IL-1 $\beta$, were significantly reduced 
by caudatin. The levels of Ki67, a mitotic marker expressed from the mid-G1 phase to the end of mitosis, were also substantially reduced by caudatin. Notably, accumulating data has indicated that $\mathrm{Ki} 67$ is involved in the regulation of mitotic progression, including chromatin organization, DNA replication and interactions with motor proteins to control centrosome separation (66). A fraction of Ki-67-positive tumor cells is often correlated with the clinical course of cancer and the tumor grade (67). A previous study observed a positive correlation between the Ki67 expression levels and the risk of local recurrence (68).

In summary, the present study demonstrated caudatin as an effective anti-hepatocarcinogenesis compound, suggesting that the anticancer effects are probably mediated by regulating the PERK-ATF4-eIF2 $\alpha$ pathway and ATF6 cleavage. Concerning the decline of GRP78 by caudatin in liver nodules, further studies are required to identify the potential of caudatin in ameliorating metastasis and chemoresistance, advancing the prognosis in HCC patients.

\section{Acknowledgements}

Not applicable.

\section{Funding}

This study was supported by the National Natural Science Foundation of China (grant nos. 81773947, 81873055, 81303275 and 81603382) and Foundation for High-Level Talent in Six Areas of Jiangsu Province (grant no. WSN-042), 'Double First-Class' University project of China Pharmaceutical University (grant nos. CPU2018GF07 and CPU2018PZQ19), Key research projects on modernization of traditional Chinese medicine (grant no. 2018YFC1706900), Medical innovation team of Jiangsu province (grant no. CXTDB2017003), Research Plan for Chinese Medicine Bureau of Jiangsu province (grant no. YB2017034). Scientific Research Project of State Traditional Chinese Medicine Clinical Base Construction (grant no. JDZX2015072), Program for Innovative Research Team of Six Talent Peaks Project in Jiangsu Province (grant no. SWYY-CXTD-004).

\section{Availability of data and materials}

The datasets used during the current study are available from the corresponding author on reasonable request.

\section{Authors' contributions}

YL and LF conceived and designed the experiments. JS, WD and XJ carried out the experiments. JS and WD drafted the manuscript. ZX and WD performed the MRI. BL and DL helped with the statistical analysis. LZ and LC helped with the ELISA analysis. JS and XJ revised the paper. All authors read and approved the final manuscript.

\section{Ethics approval and consent to participate}

All of the animal procedures, including housing, care and experimental protocols, were approved by the Animal Care and Use Committee of Affiliated Hospital of Integrated Traditional Chinese and Western Medicine.

\section{Patient consent for publication}

Not applicable.

\section{Competing interests}

The authors declare that they have no competing interests.

\section{References}

1. Wang Y, Yang Z, Wang L, Sun L, Liu Z, Li Q, Yao B, Chen T, Wang C, Yang W, et al: miR-532-3p promotes hepatocellular carcinoma progression by targeting PTPRT. Biomed Pharmacother 109: 991-999, 2019.

2. Fang D, Xiong Z, Xu J, Yin J and Luo R: Chemopreventive mechanisms of galangin against hepatocellular carcinoma: A review. Biomed Pharmacother 109: 2054-2061, 2019.

3. Shlomai A, de Jong YP and Rice CM: Virus associated malignancies: The role of viral hepatitis in hepatocellular carcinoma. Semin Cancer Biol 26: 78-88, 2014.

4. Liu J, Fan L, Yu H, Zhang J, He Y, Feng D, Wang F, Li X, Liu Q, Li Y, et al: Endoplasmic reticulum stress causes liver cancer cells to release Exosomal miR-23a-3p and Up-regulate programmed death Ligand 1 expression in macrophages. Hepatology 70: 241-258, 2019.

5. Li G, Liu D, Cooper TK, Kimchi ET, Qi X, Avella DM, Li N, Yang QX, Kester M, Rountree CB, et al: Successful chemoimmunotherapy against hepatocellular cancer in a novel murine model. J Hepatol 66: 75-85, 2017.

6. Wu K, Kryczek I, Chen L, Zou W and Welling TH: Kupffer cell suppression of CD8+ T cells in human hepatocellular carcinoma is mediated by B7-H1/programmed death-1 interactions. Cancer Res 69: 8067-8075, 2009.

7. Park EJ, Lee JH, Yu GY, He G, Ali SR, Holzer RG, Osterreicher $\mathrm{CH}$, Takahashi $\mathrm{H}$ and Karin M: Dietary and genetic obesity promote liver inflammation and tumorigenesis by enhancing IL-6 and TNF expression. Cell 140: 197-208, 2010.

8. Clarke HJ, Chambers JE, Liniker E and Marciniak SJ: Endoplasmic reticulum stress in malignancy. Cancer Cell 25: 563-573, 2014.

9. Peñaranda Fajardo NM, Meijer C and Kruyt FA: The endoplasmic reticulum stress/unfolded protein response in gliomagenesis, tumor progression and as a therapeutic target in glioblastoma. Biochem Pharmacol 118: 1-8, 2016.

10. Fan L, Sun G, Ma T, Zhong F, Lei Y, Li X and Wei W: Melatonin reverses tunicamycin-induced endoplasmic reticulum stress in human hepatocellular carcinoma cells and improves cytotoxic response to doxorubicin by increasing CHOP and decreasing survivin. J Pineal Res 55: 184-194, 2013.

11. Zha L, Fan L, Sun G, Wang H, Ma T, Zhong F and Wei W: Melatonin sensitizes human hepatoma cells to endoplasmic reticulum stress-induced apoptosis. J Pineal Res 52: 322-331, 2012.

12. Chen Y, Tsai YH and Tseng SH: HDAC Inhibitors and RECK modulate endoplasmic reticulum stress in tumor cells. Int J Mol Sci 18: pii: E258, 2017.

13. Vandewynckel YP, Laukens D, Bogaerts E, Paridaens A, Van den Bussche A, Verhelst X, Van Steenkiste C, Descamps B, Vanhove C, Libbrecht L, et al: Modulation of the unfolded protein response impedes tumor cell adaptation to proteotoxic stress: A PERK for hepatocellular carcinoma therapy. Hepatol Int 9: 93-104, 2014.

14. Li J and Lee AS: Stress induction of GRP78/BiP and its role in cancer. Curr Mol Med 6: 45-54, 2006.

15. Lebeau P, Byun JH, Yousof T and Austin RC: Pharmacologic inhibition of S1P attenuates ATF6 expression, causes ER stress and contributes to apoptotic cell death. Toxicol Appl Pharmacol 349: 1-7, 2018

16. Spaan CN, Smit WL, van Lidth de Jeude JF, Meijer BJ, Muncan V, van den Brink GR and Heijmans J: Expression of UPR effector proteins ATF6 and XBP1 reduce colorectal cancer cell proliferation and stemness by activating PERK signaling. Cell Death Dis 10: 490, 2019. 
17. Scaiewicz V, Nahmias A, Chung RT, Mueller T, Tirosh B and Shibolet O: CCAAT/enhancer-binding protein homologous (CHOP) protein promotes carcinogenesis in the DEN-induced hepatocellular carcinoma model. PLoS One 8: e81065, 2013.

18. Hetz C: The unfolded protein response: Controlling cell fate decisions under ER stress and beyond. Nat Rev Mol Cell Biol 13: 89-102, 2012

19. Shuda M, Kondoh N, Imazeki N, Tanaka K, Okada T, Mori K, Hada A, Arai M, Wakatsuki T, Matsubara O, et al: Activation of the ATF6, XBP1 and grp78 genes in human hepatocellular carcinoma: A possible involvement of the ER stress pathway in hepatocarcinogenesis. J Hepatol 38: 605-614, 2003.

20. Wang X, Fu X, Zhao S, Fu X, Zhang H, Shao L, Li G and Fan C: Antiangiogenic properties of caudatin in vitro and in vivo by suppression of VEGF-VEGFR2-AKT/FAK signal axis. Mol Med Rep 16: 8937-8943, 2017.

21. Tan ZW, Xie S, Hu SY, Liao T, Liu P, Peng KH, Yang XZ, He ZL, Tang HY, Cui Y, et al: Caudatin targets TNFAIP1/NF- $\kappa$ B and cytochrome c/caspase signaling to suppress tumor progression in human uterine cancer. Int J Oncol 49: 1638-1650, 2016.

22. Peng Y and Ding Y: Pharmacokinetics and tissue distribution study of caudatin in normal and diethylnitrosamine-induced hepatocellular carcinoma model rats. Molecules 20: 4225-4237, 2015.

23. Fu XY, Zhang S, Wang K, Yang MF, Fan CD and Sun BL: Caudatin inhibits human Glioma cells growth through triggering DNA damage-mediated cell cycle arrest. Cell Mol Neurobiol 35: 953-959, 2015

24. Li X, Zhang X, Liu X, Tan Z, Yang C, Ding X, Hu X, Zhou J, Xiang S, Zhou $\mathrm{C}$ and Zhang J: Caudatin induces cell apoptosis in gastric cancer cells through modulation of Wnt/beta-catenin signaling. Oncol Rep 30: 677-684, 2013.

25. Luo Y, Sun Z, Li Y, Liu L, Cai X and Li Z: Caudatin inhibits human hepatoma cell growth and metastasis through modulation of the Wnt//-catenin pathway. Oncol Rep 30: 2923-2928, 2013.

26. Livak KJ and Schmittgen TD: Analysis of relative gene expression data using real-time quantitative PCR and the 2(-Delta Delta C(T)) method. Methods 25: 402-408, 2001.

27. Bakiri L, Hamacher R, Graña O, Guío-Carrión A, Campos-Olivas R, Martinez L, Dienes HP, Thomsen MK, Hasenfuss SC and Wagner EF: Liver carcinogenesis by FOS-dependent inflammation and cholesterol dysregulation. J Exp Med 214: 1387-1409, 2017.

28. Liang S, Ma HY, Zhong Z, Dhar D, Liu X, Xu J, Koyama Y, Nishio T, Karin D, Karin G, et al: NADPH oxidase 1 in liver macrophages promotes inflammation and tumor development in mice. Gastroenterology 156: 1156-1172.e6, 2019.

29. Mahmoud AM, Mohammed HM, Khadrawy SM and Galaly SR Hesperidin protects against chemically induced hepatocarcinogenesis via modulation of Nrf2/ARE/HO-1, PPAR $\gamma$ and TGF- $31 / \mathrm{Smad} 3$ signaling, and amelioration of oxidative stress and inflammation. Chem Biol Interact 277: 146-158, 2017.

30. Lu JW, Liao CY, Yang WY, Lin YM, Jin SL, Wang HD and Yuh CH: Overexpression of endothelin 1 triggers hepatocarcinogenesis in zebrafish and promotes cell proliferation and migration through the AKT pathway. PLoS One 9: e85318, 2014

31. Kobylewski SE, Henderson KA, Yamada KE and Eckhert CD Activation of the EIF2 $\alpha /$ ATF4 and ATF6 Pathways in DU-145 Cells by boric acid at the concentration reported in men at the US mean boron intake. Biol Trace Elem Res 176: 278-293, 2017.

32. Shoulders MD, Ryno LM, Genereux JC, Moresco JJ, Tu PG, Wu C, Yates JR III, Su AI, Kelly JW and Wiseman RL: Stress-independent activation of XBP1s and/or ATF6 reveals three functionally diverse ER proteostasis environments. Cell Rep 3: 1279-1292, 2013

33. Lee AH, Iwakoshi NN and Glimcher LH: XBP-1 regulates a subset of endoplasmic reticulum resident chaperone genes in the unfolded protein response. Mol Cell Biol 23: 7448-7459, 2003

34. Tolba R, Kraus T, Liedtke C, Schwarz M and Weiskirchen R: Diethylnitrosamine (DEN)-induced carcinogenic liver injury in mice. Lab Anim 49 (Suppl 1): S59-S69, 2015.

35. Lin $\mathrm{H}$, Liu XB, Yu JJ, Hua F and $\mathrm{Hu} \mathrm{ZW}$ : Antioxidant $\mathrm{N}$-acetylcysteine attenuates hepatocarcinogenesis by inhibiting ROS/ER stress in TLR2 deficient mouse. PLoS One 8: e74130, 2013.

36. Liu H, Zhang X, Zhang S, Huang H, Wu J, Wang Y, Yuan L, Liu C, Zeng X, Cheng X, et al: Oxidative stress mediates Microcystin-LR-induced endoplasmic reticulum stress and autophagy in KK-1 cells and C57BL/6 mice ovaries. Front Physiol 9: 1058, 2018
37. Liu M, Du L, He Z, Yan L, Shi Y, Shang J and Tang H: Increased ERp57 Expression in HBV-related hepatocellular carcinoma: Possible correlation and prognosis. Biomed Res Int 2017: $1252647,2017$.

38. Zhou X, Han D, Yang X, Wang X and Qiao A: Glucose regulated protein 78 is potentially an important player in the development of nonalcoholic steatohepatitis. Gene 637: 138-144, 2017.

39. Nermin AHS,EL-MaraghySA andManalFI:Diethylnitrosamineinduced hepatocarcinogenesis in rats: Possible chemoprevention by blueberries. African J Bioch Res 2: 81-87, 2008.

40. Wang P, Ji R, Ji J and Chen F: Changes of metabolites of acrylamide and glycidamide in acrylamide-exposed rats pretreated with blueberry anthocyanins extract. Food Chem 274: 611-619, 2019.

41. Ghosh S, Nandi S, Ghosh C and Bhattacharyya K: Fluorescence dynamics in the endoplasmic reticulum of a live cell: Time-resolved confocal microscopy. Chemphyschem 17: 2818-2823, 2016

42. Huang RZ, Huang XC, Zhang B, Jia HY, Liao ZX and Wang HS: 16-O-caffeoyl-16-hydroxylhexadecanoic acid, a medicinal plant-derived phenylpropanoid, induces apoptosis in human hepatocarcinoma cells through ROS-dependent endoplasmic reticulum stress. Phytomedicine 41: 33-44, 2018

43. Wang $M$ and Kaufman RJ: The impact of the endoplasmic reticulum protein-folding environment on cancer development. Nat Rev Cancer 14: 581-597, 2014.

44. Wakai T, Zhang N, Vangheluwe P and Fissore RA: Regulation of endoplasmic reticulum $\mathrm{Ca}(2+)$ oscillations in mammalian eggs J Cell Sci 126: 5714-5724, 2013.

45. Cubillos-Ruiz JR, Bettigole SE and Glimcher LH: Tumorigenic and immunosuppressive effects of endoplasmic reticulum stress in cancer. Cell 168: 692-706, 2017.

46. Tang H, Bao X, Shen Y, Song M, Wang S, Wang C and Hou J: Engineering protein folding and translocation improves heterologous protein secretion in Saccharomyces cerevisiae. Biotechnol Bioeng 112: 1872-1882, 2015.

47. Li X, Zhang K and Li Z: Unfolded protein response in cancer: The physician's perspective. J Hematol Oncol 4: 8, 2011.

48. Jiang CC, Yang F, Thorne RF, Zhu BK, Hersey P and Zhang XD Human melanoma cells under endoplasmic reticulum stress acquire resistance to microtubule-targeting drugs through XBP-1-mediated activation of Akt. Neoplasia 11: 436-447, 2009.

49. Pfaffenbach KT and Lee AS: The critical role of GRP78 in physiologic and pathologic stress. Curr Opin Cell Biol 23: 150-156, 2011.

50. Kao RH, Lai GM, Chow JM, Liao CH, Zheng YM, Tsai WL, Hsia S, Lai IC, Lee HL, Chuang SE, et al: Opposite regulation of CHOP and GRP78 and synergistic apoptosis induction by selenium Yeast and Fish Oil via AMPK activation in lung adenocarcinoma cells. Nutrients 10: pii: E1458, 2018

51. Liu R, Li X, Gao W, Zhou Y, Wey S, Mitra SK, Krasnoperov V, Dong D, Liu S, Li D, et al: Monoclonal antibody against cell surface GRP78 as a novel agent in suppressing PI3K/AKT signaling, tumor growth, and metastasis. Clin Cancer Res 19: 6802-6811, 2013

52. Hensel F, Eckstein M, Rosenwald A and Brandlein S: Early development of PAT-SM6 for the treatment of melanoma. Melanoma Res 23: 264-275, 2013.

53. Kanemoto S, Nitani R, Murakami T, Kaneko M, Asada R, Matsuhisa K, Saito A and Imaizumi K: Multivesicular body formation enhancement and exosome release during endoplasmic reticulum stress. Biochem Biophys Res Commun 480: 166-172, 2016.

54. Zuo L, Zhu Y, Hu L, Liu Y, Wang Y, Hu Y, Wang H, Pan X, Li K, Du N and Huang Y: PI3-kinase/Akt pathway-regulated membrane transportation of acid-sensing ion channel 1a/Calcium ion influx/endoplasmic reticulum stress activation on PDGF-induced HSC Activation. J Cell Mol Med 23: 3940-3950, 2019.

55. Schonthal AH: Pharmacological targeting of endoplasmic reticulum stress signaling in cancer. Biochem Pharmacol 85: 653-666, 2013

56. Hamanaka RB, Bennett BS, Cullinan SB and Diehl JA: PERK and GCN2 contribute to eIF2alpha phosphorylation and cell cycle arrest after activation of the unfolded protein response pathway. Mol Biol Cell 16: 5493-5501, 2005.

57. Sanchez-Alvarez M, Del Pozo MA and Bakal C: Publisher correction: AKT-mTOR signaling modulates the dynamics of IRE1 RNAse activity by regulating ER-mitochondria contacts. Sci Rep 8: 6476, 2018. 
58. Zhang Z, Chen J, Chen F, Yu D, Li R, Lv C, Wang H, Li H, Li J and Cai Y: Tauroursodeoxycholic acid alleviates secondary injury in the spinal cord via up-regulation of CIBZ gene. Cell Stress Chaperones 23: 551-560, 2018.

59. Bettigole SE and Glimcher LH: Endoplasmic reticulum stress in immunity. Ann Rev Immunol 33: 107-138, 2015.

60. Verfaillie T, Garg AD and Agostinis P: Targeting ER stress induced apoptosis and inflammation in cancer. Cancer Lett 332: 249-264, 2013

61. Zhang K and Kaufman RJ: From endoplasmic-reticulum stress to the inflammatory response. Nature 454: 455-462, 2008.

62. Taniguchi K and Karin M: IL-6 and related cytokines as the critical lynchpins between inflammation and cancer. Semin Immunol 26: 54-74, 2014

63. Nakagawa H, Umemura A, Taniguchi K, Font-Burgada J, Dhar D, Ogata H, Zhong Z, Valasek MA, Seki E, Hidalgo J, et al: ER stress cooperates with hypernutrition to trigger TNF-dependent spontaneous HCC development. Cancer Cell 26: 331-343, 2014.

64. Xiang DM, Sun W, Ning BF, Zhou TF, Li XF, Zhong W, Cheng Z, Xia MY, Wang X, Deng X, et al: The HLF/IL-6/STAT3 feedforward circuit drives hepatic stellate cell activation to promote liver fibrosis. Gut 67: 1704-1715, 2018.
65. Gosain R, Anwar S, Miller A, Iyer R and Mukherjee S: Interleukin- 6 as a biomarker in patients with hepatobiliary cancers. J Gastrointest Oncol 10: 537-545, 2019.

66. Cuylen S, Blaukopf C, Politi AZ, Müller-Reichert T, Neumann B, Poser I, Ellenberg J, Hyman AA and Gerlich DW: Ki-67 acts as a biological surfactant to disperse mitotic chromosomes. Nature 535: 308-312, 2016.

67. Basturk O, Yang Z, Tang LH, Hruban RH, Adsay V, McCall CM, Krasinskas AM, Jang KT, Frankel WL, Balci S, et al: The high-grade (WHO G3) pancreatic neuroendocrine tumor category is morphologically and biologically heterogenous and includes both well differentiated and poorly differentiated neoplasms. Am J Surg Pathol 39: 683-690, 2015.

68. Inwald EC, Klinkhammer-Schalke M, Hofstadter F, Zeman F, Koller M, Gerstenhauer M and Ortmann O: $\mathrm{Ki}-67$ is a prognostic parameter in breast cancer patients: Results of a large population-based cohort of a cancer registry. Breast Cancer Res Treat 139: 539-552, 2013.

(i) (5) $\ominus$ This work is licensed under a Creative Commons Attribution-NonCommercial-NoDerivatives 4.0 International (CC BY-NC-ND 4.0) License. 